\title{
Turbulence et diffusion d'un gaz lourd dans l'atmosphère
}

\section{Turbulence and diffusion of a heavy gas inside the atmosphere}

\author{
D. Grand \\ J.F. Fourmigue \\ M. Villand
}

Commissariat à l'énergie atomique Centre d'études nucléaires de Grenoble

L'émission dans l'atmosphère d'un gaz polluant ou inflammable est un des risques potentiels de l'industrie chimique. L'étude décrite a été entreprise en vue de prédire l'évolution d'un gaz lourd près de son point d'émission. Elle a été réalisée à l'aide du système TRIO de logiciels de calcul d'écoulements turbulents tridimensionnels, destiné, à l'origine, au calcul des phénomènes thermohydrauliques mis en jeu dans les installations nucléaires.

La variété des phénomènes présents dans ces installations a conduit à la réalisation de logiciels de. résolution de problèmes thermohydrauliques généraux : un logiciel fondé sur la méthode des éléments finis (TRIO.EF), un logiciel fondé sur la méthode des volumes finis (TRIO.VF). Ils utilisent une modélisation de la turbulence du type $(k, \varepsilon)$ avec prise en compte des effets de densité.

Les prédictions du logiciel TRIO.VF sont comparées avec des essais de rejet d'un gaz lourd réalisés sur le site de Thorney Island dans le cadre d'un programme européen.

The dispersion of a toxic or flammable gas into the atmosphere is one of the potential risks of the chemical industry. The present study deals with the transport of a heavy gas in the atmosphere. A numerical simulation carried out with the TRIO system which was developed for multidimensional flow computations is presented.

The TRIO system was originally developed for computation of thermal hydraulic phenomena in the nuclear industry - General methods had to be used to deal with the variety of flow phenomena encountered - Two solvers were developed based on a finite element methode $(E F)$ and a finite volume method (VF).

The results of TRIO-VF are compared to the heavy gas dispersion experiments on Thorney Island.

L'émission dans l'atmosphère d'un gaz polluant ou inflammable est un des risques potentiels de l'industrie chimique. En liaison avec le C.E.A./IPSN, une étude a été entreprise en vue de prédire l'évolution d'un gaz lourd près de son point d'émission.

L'étude a été réalisée à l'aide du système TRIO de logiciels de calcul d'écoulements turbulents tridimensionnels, destiné, à l'origine, au calcul des phénomènes ther- mohydrauliques mis en jeu dans les installations nucléaires. La variété des phénomènes présents dans ces installations a conduit à la réalisation de logiciels de résolution de problèmes thermohydrauliques généraux : un logiciel fondé sur la méthode des éléments finis (TRIO.EF), un logiciel fondé sur la méthode des volumes finis (TRIO.VF). Ils utilisent une modélisation de la turbulence du type $(k, \varepsilon)$ avec prise en compte des effets de densité. 

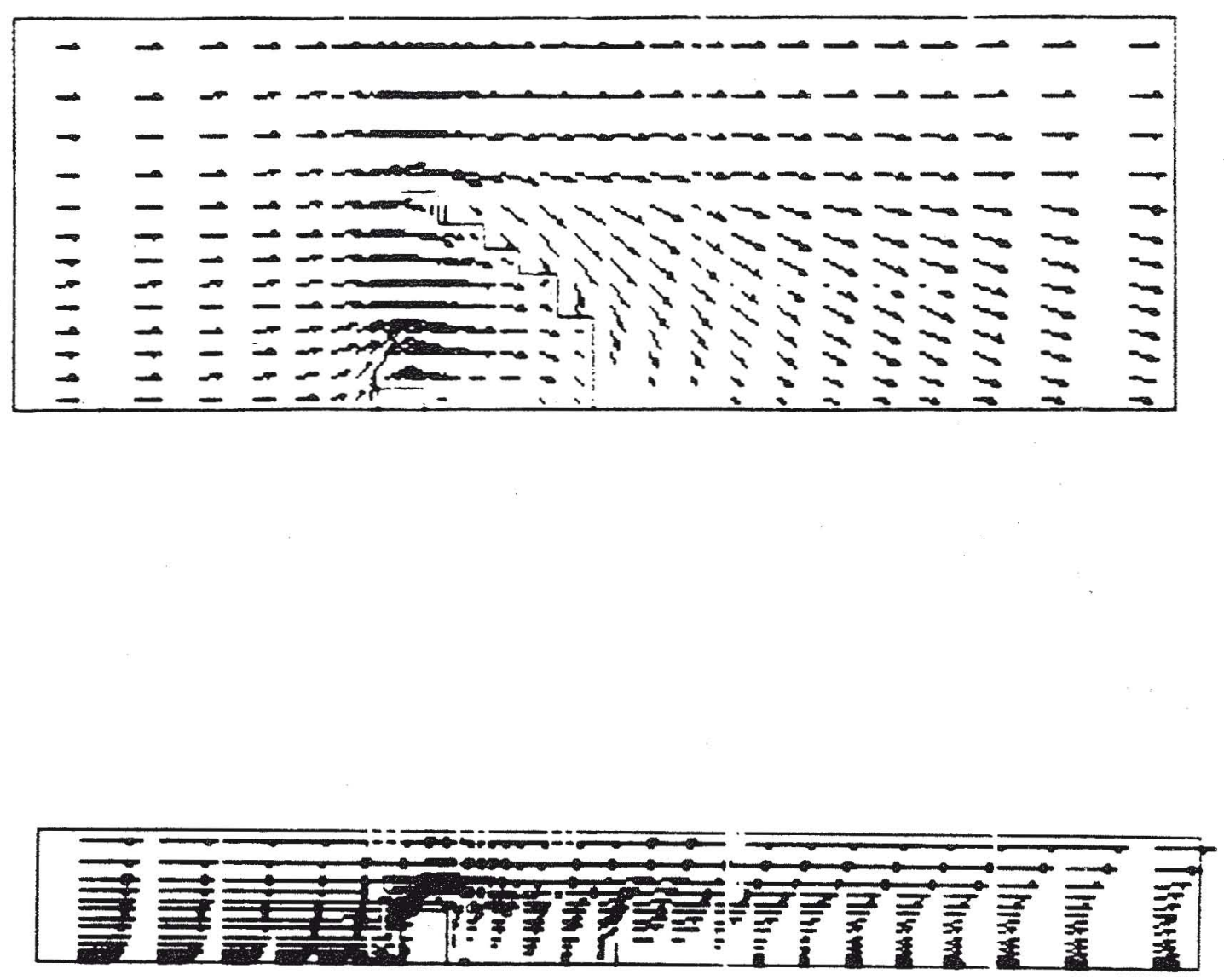

1. Régime hydraulique établie.

Dans l'exemple qui suit, on compare les prédictions du logiciel TRIO.VF avec des essais de rejet d'un gaz lourd réalisés sur le site de Thorney Island dans le cadre d'un programme européen.

Dans ces expériences, $2000 \mathrm{~m}^{3}$ de gaz sont contenus dans une tente cylindrique. Un mur semi-circulaire est placé en aval de la tente. L'écoulement initial et les recirculations induites par les obstacles sont montrés en figure $l$ en vues de dessus et de profil. Le calcul est fait dans le demi espace délimité par le plan vertical formé par la direction du vent et l'axe de la tente.

A l'instant initial, les parois tombent mettant le gaz en contact avec l'atmosphère. On observe l'évolution du nuage de gaz. Les figures 2 (vue de dessus) et 3 (vue de profil) montrent les évolutions des concentrations avec le temps. 

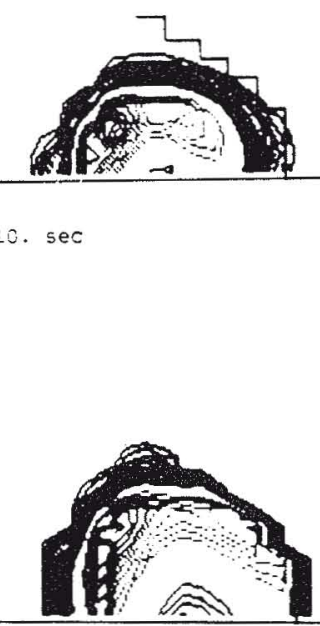

$t=20 . \mathrm{sec}$

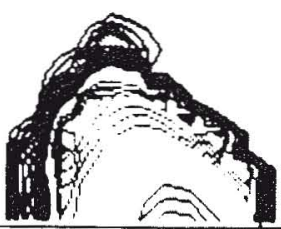

$t=30 \cdot \sec$
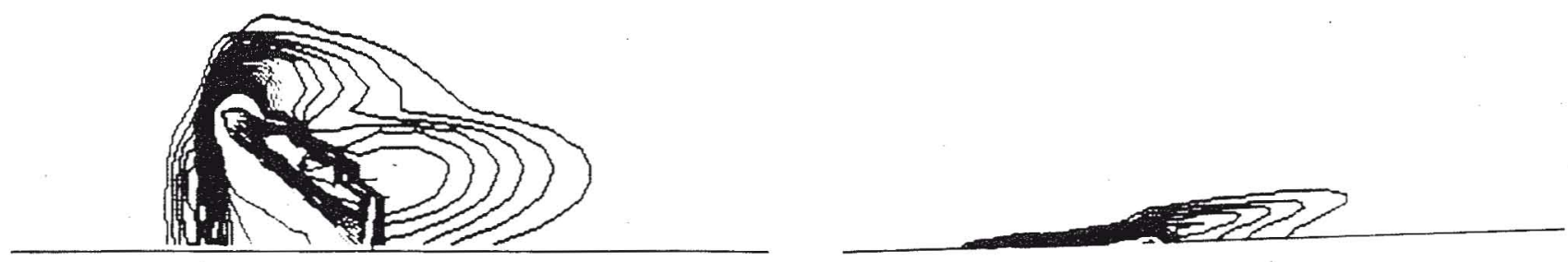

$t=50 . \mathrm{sec}$
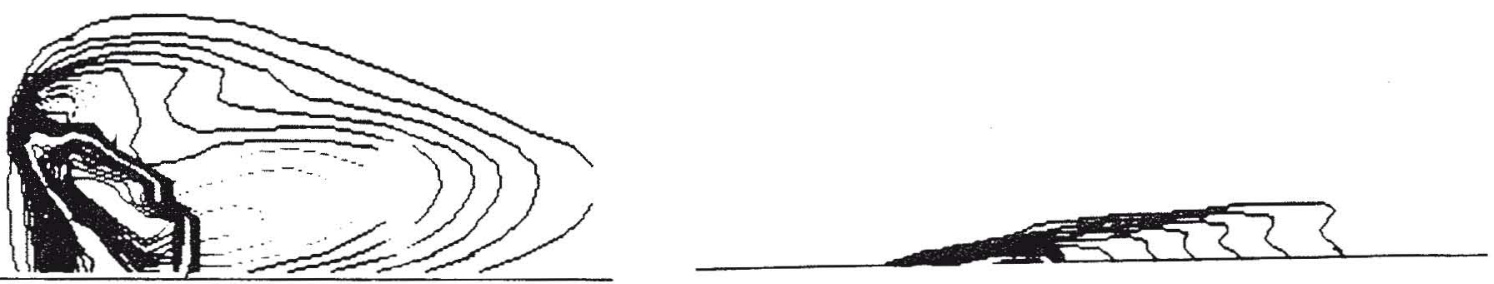

$==80 . \mathrm{sec}$

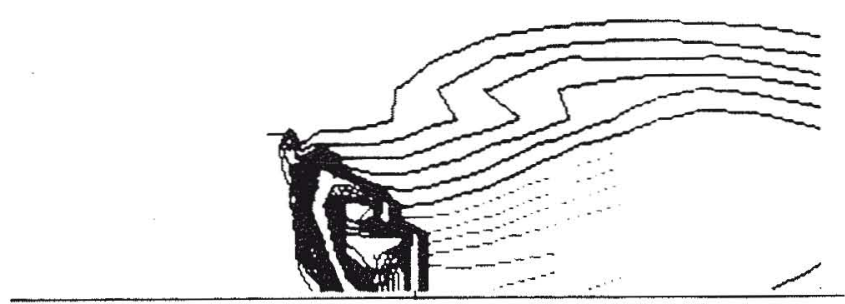

$t=: 20 . \mathrm{sec}$ 

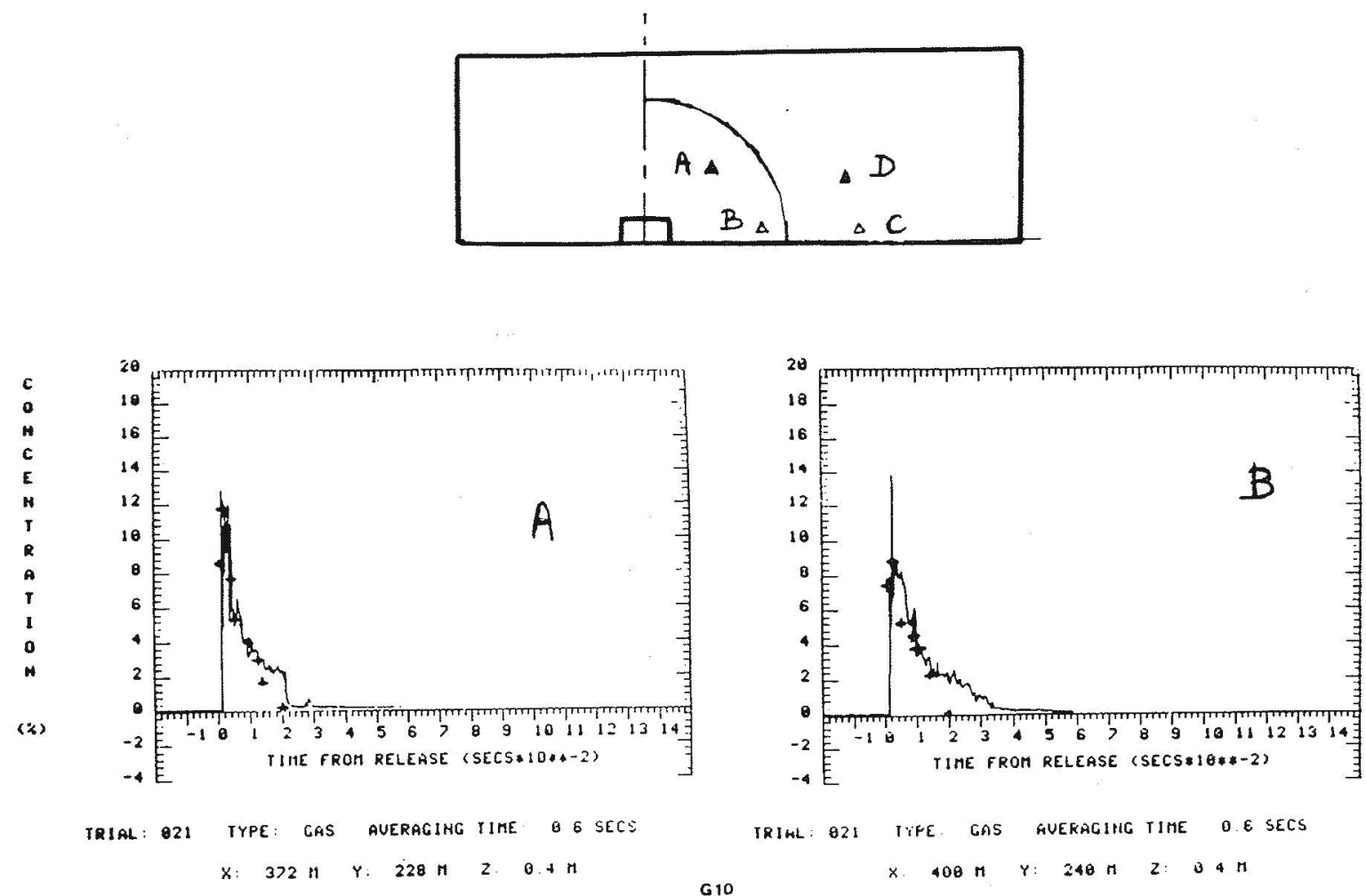

IRIHL: 021 TIFE GAS GUERAGIHG TIME O.E SECS
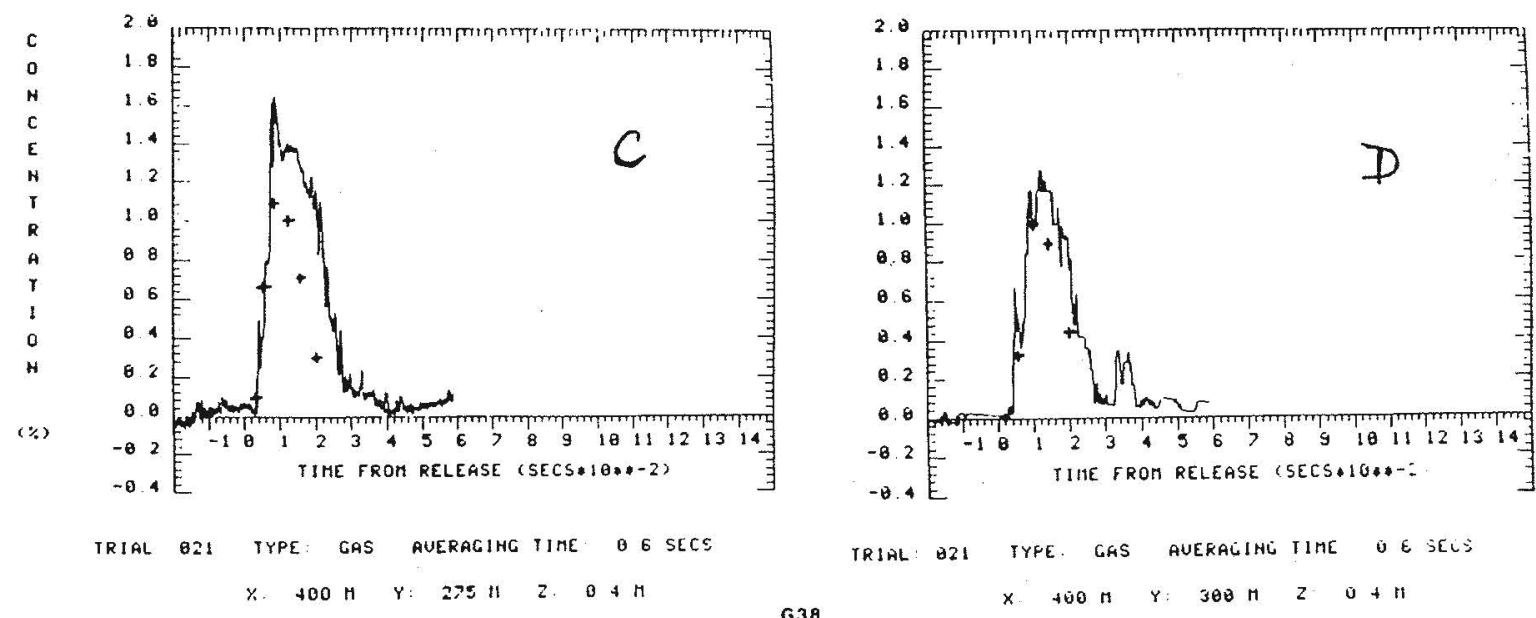

TRIAL OZI TYFE. GGS AUERALINL TIML OE SELS

4.

La figure 4 présente des comparaisons entre les concentrations calculées et celles relevées sur des sondes fixes.

L'analyse des rejets accidentels de gaz polluant constitue un champ nouveau d'applications qui touchent au domaine nucléaire : accident chimique au voisinage d'une installation nucléaire, contamination d'un local... Leur utilisation dès la conception des installations peut permettre l'optimisation de ces installations du point de vue des critères économiques et de sûreté, de façon beaucoup plus approfondie que par le seul recours aux expériences physiques.

Adresse des auteurs

Messieurs D. Grand, J.F. Fourmigue et $M$. Villand Centre d'études nucléaires de Grenoble,

Service des Transferts thermiques,

Laboratoire de physique, modélisation et logiciels, $85 X$ avenue des Martyrs, 38041 Grenoble Cedex. 